\title{
GPC-3 in hepatocellular carcinoma: current perspectives
}

This article was published in the following Dove Press journal:

Journal of Hepatocellular Carcinoma

8 November 2016

Number of times this article has been viewed

\author{
Yongle $\mathrm{Wu}^{\prime}$ \\ Hui Liu² \\ Huiguo Ding' \\ 'Department of Gastroenterology \\ and Hepatology, ${ }^{2}$ Department of \\ Pathology, Beijing You'an Hospital, \\ Affiliated with Capital Medical \\ University, Beijing , People's Republic \\ of China
}

\begin{abstract}
Glypican-3 (GPC3), a member of heparan sulfate proteoglycans, attaches to the cell membrane and is frequently observed to be elevated in hepatocellular carcinoma (HCC). However, GPC3 is not detected in normal liver tissues and benign liver lesions. Consequently, GPC3 is currently being used as a diagnostic biomarker and HCC-specific positron emission computed tomography probe to identify HCCs in normal liver tissues and benign liver lesions. The overexpression of GPC-3 in serum or liver tissue also predicts poor prognosis for HCC patients. In addition, GPC3 promotes HCC growth and metastasis by activating the canonical Wnt and other signaling pathways. Targeting of GPC3, including GC33, HN3 and YP7, might offer new immunotherapeutic tools for HCC treatment.
\end{abstract}

Keywords: glypican-3, hepatocellular carcinoma, diagnostics, prognosis, immunotherapy

\section{Introduction}

Hepatocellular carcinoma (HCC) is one of the most common malignant tumors and the third leading cause of cancer-related death in the world. ${ }^{1}$ Although a great progress has been made in $\mathrm{HCC}$ treatment, including curative surgery and nonsurgical treatment, the HCC prognosis remains poor. ${ }^{1}$ The result of treatment depends on the HCC stage at the time of diagnosis. HCC can be cured if diagnosed at an early stage. However, most HCCs are diagnosed at an advanced stage when patients visit physicians with symptoms.

Glypican-3 (GPC3), a member of the heparan sulfate (HS) proteoglycan family, is attached to the cell surface by a glycosyl-phosphatidylinositol (GPI) anchor and can be cleaved off the cell surface. The soluble GPC3 can be detected in serum (sGPC3). ${ }^{2,3}$ GPC3 is widely expressed in human embryos and plays a significant role in morphogenesis and growth, by mechanisms involving insulin-like growth factor, bone morphogenetic protein (BMP), fibroblast growth factor (FGF) or hedgehog (Hh) signaling pathway. ${ }^{4-6}$ GPC3 can be detected in the fetal liver from embryonic weeks 18 to 30 , but cannot be identified in any normal adult hepatic tissue. ${ }^{4-7}$ In recent years, extensive research has been carried out on the role of GPC3 in diagnosis, progression and treatment of $\mathrm{HCC}$ in vivo and in vitro. ${ }^{8,9}$ Here, we summarize current evidences for the use of GPC3 as a diagnostic biomarker, its oncogenic function and as a immunotherapeutic target for HCC patients.
Correspondence: Huiguo Ding Department of Gastroenterology and Hepatology, Beijing You'an Hospital, Affiliated with Capital Medical University, No.8 Yoanmen Wai Street, Fengtai District, Beijing 100069, China

Tel +861083997155

Fax +861063295525

Email dinghuiguo@medmail.com.cn 


\section{GPC3 as a biomarker for diagnosis and prognosis of $\mathrm{HCC}$}

In 1997, Hsu et $\mathrm{al}^{7}$ first reported that mRNA and protein levels of GPC 3 were upregulated to a greater extent in most HCCs than in normal liver, cholangiocarcinoma and metastatic carcinomas of the liver. Since then, the diagnostic value of GPC3 in HCC has been studied extensively, and increasing studies have confirmed that GPC 3 would be a useful serological and immunohistochemical biomarker for HCC. By immunolabeling GPC 3 with a monoclonal antibody, Capurro et al ${ }^{8}$ revealed that $72 \%$ of HCCs were GPC3-positive; however, GPC3 was undetectable in normal liver tissues, cirrhosis or benign lesions. Other studies conducted on GPC 3 across the world also revealed similar results. ${ }^{9-13}$ Besides, the membranebound GPC 3 can also be cleaved off by lipase from the GPI anchor. ${ }^{14}$ Thus, the diagnostic value of sGPC 3 was evaluated later by several studies. Our study also verified that GPC3 was a sensitive and specific biomarker for diagnosis of early HCC due to its high expression in HCC tissue. ${ }^{9}$ Moreover, we found that sGPC3 was detectable in $48.8 \%$ of patients with negative serum $\alpha$-fetoprotein (AFP), which further confirmed that GPC3 might be a serum marker for $\mathrm{HCC}$ and indicated that GPC3 could be used to distinguish AFP-negative HCC from cirrhotic nodules. ${ }^{11}$ Qiao et al analyzed the serum levels of GPC3, AFP and human cervical cancer oncogene (HCCR) in 189 cases (101 HCC, 40 cirrhosis, 18 hepatitis and 30 control healthy donors).${ }^{10}$ They concluded that GPC 3 was the best sensitive biomarker of the three biomarkers mentioned earlier, and the combination of these three biomarkers, with a sensitivity of $80.2 \%$, was much higher than AFP alone. ${ }^{11}$ In recent years, in vivo and in vitro studies displayed the use of Zr- $\alpha$ GPC3, a HCC-specific positron emission computed tomography (PET) probe, in HCC imaging as well as the detection of GPC3 levels by small-animal PET. Those results suggested that the identification of small liver lesions with a HCC-specific PET probe would help physicians to make the differential diagnosis between HCCs and benign lesions in cirrhotic patients so that patient management could be significantly altered..$^{13,14}$ In addition, extensive studies to determine the prognostic value of GPC3 were carried out in $\mathrm{HCC}$ patients. ${ }^{15-19}$ Increasing evidence revealed that high GPC3 expression was a prominent prognostic factor that predicted a poor outcome for HCCs. Clinicopathological studies on GPC3-Immunohistochemistry stainings revealed that high GPC3 expression was associated with poor postoperative disease-free survival (DFS) and overall survival (OS) and that it also served as an independent risk factor. ${ }^{15-17}$ Patients with overexpressed GPC3 in HCC tissues showed notably shorter OS and DFS than those with underexpressed GPC3. Several meta-analytic studies also supported the fact that a high GPC3-IHC score was one of the prognostic factors in HCC, as there was a significantly negative correlation between GPC3 expression and OS or DFS of HCC patients. ${ }^{18,19}$

\section{GPC3-mediated signaling pathway in HCC progression}

In addition to being a reliable indicator for the diagnosis and prognosis of $\mathrm{HCC}, \mathrm{GPC} 3$ has a significant role in the progression of HCC (Figure 1). GPC3 functions as a coreceptor/storage site for some ligands, e.g. Wnt and FGF, via its HS side chains, and facilitates ligand and/or its receptors to stimulate the signaling pathways involved in HCC growth and invasion. Several studies showed that GPC3 could promote the growth of hepatoma cells in vivo and in vitro. ${ }^{20-23}$ Capurro et al suggested that GPC3 promoted the growth of hepatoma cells by stimulating canonical $\mathrm{Wnt} / \beta$-catenin signaling pathway activity in vivo and in vitro. ${ }^{20}$ Recently, this GPC3-mediated activation of Wnt signaling pathway in HCC cells has been confirmed by other reports. ${ }^{21,22} \mathrm{Li}$ et al showed that ectopic GPC 3 could increase the c-Myc expression, a typical target of the canonical Wnt signaling pathway; c-Myc can also transcriptionally activate GPC3 directly in HCC cells. ${ }^{21}$ Zittermann et al ${ }^{22}$ reported that soluble GPC3, which is cleaved off the cell membrane at the GPI anchor domain, could inhibit the in vivo growth of HCC cells by blocking the canonical Wnt signaling pathway in tumors generated by Huh6 and Huh7, and Akt and ERK signaling activation in HepG2- and Huh7-derived tumors, respectively. In other words, this activity can be observed only when GPC3 is attached to the cell membrane. On the other hand, it is possible that GPC3 promotes the growth of HCC cells by mediating other signaling pathways. For example, Sun et al proved that suppression of GPC3 inhibited cell proliferation and enhanced apoptosis via upregulation of TGF- $\beta 2$ in vivo and in vitro. ${ }^{23}$ In addition, it has been demonstrated that several FGF members (FGF8, FGF17 and FGF18) were upregulated in the great majority of HCC samples. ${ }^{24}$

Metastasis is an important aspect of HCC progression, and epithelial-mesenchymal transition (EMT) is considered as the first step in the metastatic cascade. ${ }^{25}$ Recently, we have found that the expression of GPC3 in HCC tissue was upregulated during $\mathrm{HCC}$ progression from Barcelona Clinic Liver Cancer stage A or B to stage C. The increased expression of GPC 3 in tumor tissues was closely related to the level 


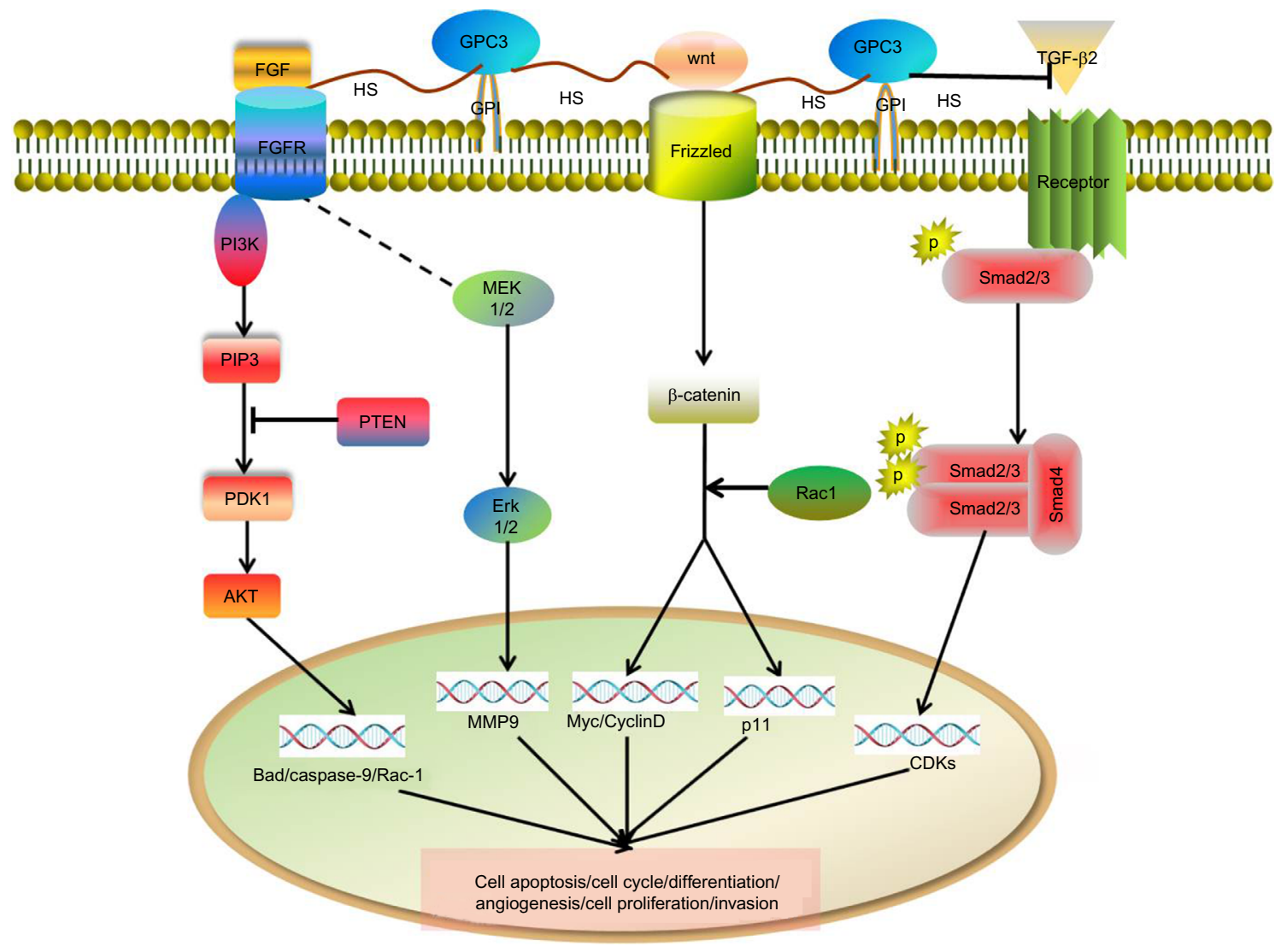

Figure I The diagram of possible GPC3-mediated signaling pathway in HCC progression. Abbreviations: GPC-3, glypican-3; HCC, hepatocellular carcinoma; P, Phosphorylation.

of EMT markers, as well as to the cancer vascular invasion. HepG2 cells, expressing a higher level of GPC3, possessed stronger ability of invasion and exhibited more EMT-like changes than those of HCC cell lines that expressed lower levels of GPC3 (Hep3B and Huh7). Our studies suggested that GPC3 promoted HCC progression and metastasis by inducing EMT in tumor cells, and the ERK signaling pathway is involved in this GPC3-induced process. ${ }^{26}$ In addition, Ruan et al also reported that GPC 3 promoted the metastasis of $\mathrm{HCC}$ in vitro and in vivo. ${ }^{27}$

\section{GPC3 is a new therapeutic target for HCC}

Based on the HCC-specific expression of GPC3 in liver, it is an emerging target for liver cancer therapy. A number of studies demonstrated that GPC3 is a potential therapeutic target for HCC. ${ }^{28-30}$ Currently, antibodies targeting GPC3, including human antibody $\mathrm{HN} 3$ and humanized mouse antibodies YP7 and GC33, are in different stages of preclinical or clinical development. GC33, a humanized mouse antibody recognizing a C-terminal domain of GPC3, showed notable cytotoxic activity against GPC3-positive hepatoma cells in vivo by complement-dependent cytotoxicity and/or antibody-dependent cell cytotoxicity. ${ }^{31-34}$ GC33 exhibited marked effect against metastatic or advanced HCC in a phase I trial, ${ }^{35}$ and the patients well tolerated a dose escalation of GC33 (2.5-20 mg/kg). ${ }^{36}$ Currently, more clinical trials for GC33 alone (phase II clinical trials) and GC33 combined with sorafenib, a chemodrug, are recruiting volunteers (phase I clinical trials). A randomized phase II clinical trial on GC33 was conducted in 185 patients with HCC metastasis. In this clinical trial, the dose of GC33 was set at 1,600 mg (intravenous) on days 1 and 8 , and then every 2 weeks thereafter. There was no significant change in mean progression-free survival (PFS) between GC33 and placebo groups (2.6 mo vs $1.5 \mathrm{mo}, \mathrm{HR}=0.97, P=0.87) .{ }^{37}$ However, higher exposure of GC33 with FcgR3A-158V polymorphism or CD16 expression intensity may prolong PFS, and therefore, further studies to analyze the GPC3-positive HCC immuno-microenvironment are necessary. ${ }^{37} \mathrm{YP7}$, a new humanized mouse anti-GPC3 antibody, has high affinity and recognizes the $\mathrm{C}$-terminal epitope that overlaps the GC33-binding site and displays the capacity of suppressing tumor activity in vivo. $\mathrm{HN} 3$, a human singledomain antibody, can also inhibit HCC cell lines and growth 
of xenograft tumors by binding to the GPC3 N-terminal and C-terminal domains. ${ }^{38}$ Currently, the GAO study group found that treatment with GPC3 monoclonal antibodies, such as HN3 and YP7, could suppress the growth of HepG2- and Hep3B-generated tumor xenografts. ${ }^{39}$ Treatment with HN3 showed higher antitumor activity than YP7..$^{40}$ Both HN3 and YP7 exhibit antitumor activity in vitro and in vivo, but their efficacies in HCC patients need to be determined and require further clinical trials.

GPC3 cannot play a dominant role in the apoptosis of HCC cells, and the fact that the GC33 or HN3 antibody could completely eliminate HCC cells needs further trials for confirmation. In combination with chemotherapy, armed antibodies, such as antibody-drug conjugates, bispecific antibodies and chimeric antigen receptor T-cell adoptive therapy may be better potential ways for HCC therapy. ${ }^{39}$

\section{Conclusion}

GPC3 is overexpressed in most of the HCC tumors, and was also used as an indicator for HCC diagnosis and prognosis. It is used as a potential target for developing therapeutic antibodies for HCC treatment. However, the relationship between GPC3 structure and function remains unclear. In addition, in order to develop GPC3-targeted therapies in HCC treatment, the expression and regulation of GPC3 in HCC need further confirmation.

\section{Acknowledgments}

The authors would like to thank Ms Shan Shan Wang for her support in providing a summary of possible signaling networks for GPC3. This study was supported by the Capital Science and Technology Development Fund (2014-1-2181), Beijing Municipal Administration of Hospitals Clinical Medicine Development of Special Funding (ZYLX201610) and Beijing Municipal Administration of Hospitals' Ascent Plan (DFL20151602).

\section{Disclosure}

The authors report no conflicts of interest in this work.

\section{References}

1. Zhu RX, Seto WK, Lai CL, Yuen MF. Epidemiology of hepatocellular carcinoma in the Asia-Pacific region. Gut Liver. 2016;10(3): 332-339.

2. Filmus J, Capurro M, Rast J. Glypicans. Genome Biol. 2008;9(5):224.

3. Traister A, Shi W, Filmus J. Mammalian Notum induces the release of glypicans and other GPI anchored proteins from the cell surface. Biochem J. 2008;410(3):503-511.

4. Yamauchi N, Watanabe A, Hishinuma M, et al. The glypican 3 oncofetal protein is a promising diagnostic marker for hepatocellular carcinoma. Mod Pathol. 2005;18(12):1591-1598.
5. Capurro MI, Xu P, Shi W, Li F, Jia A, Filmus J. Glypican-3 inhibits Hedgehog signaling during development by competing with patched for Hedgehog binding. Dev Cell. 2008;14(5):700-711.

6. Zhang L, Liu H, Sun L, Li N, Ding H, Zheng J. Glypican-3 as a potential differential diagnosis marker for hepatocellular carcinoma: a tissue microarray-based study. Acta Histochem. 2012;114(6):547-552.

7. Hsu HC, Cheng W, Lai PL. Cloning and expression of a developmentally regulated transcript MXR7 in hepatocellular carcinoma: biological significance and temporospatial distribution. Cancer Res. 1997;57(22):5179-5184.

8. Capurro M, Wanless IR, Sherman M, et al. Glypican-3: a novel serum and histochemical marker for hepatocellular carcinoma. Gastroenterology. 2003;125(1):89-97.

9. Liu H,LiP,ZhaiY, et al. Diagnostic value of glypican-3 in serum and liver for primary hepatocellular carcinoma. World J Gastroenterol. 2010;16(35): 4410-4415.

10. Qiao SS, Cui ZQ, Gong L, et al. Simultaneous measurements of serum AFP, GPC-3 and HCCR for diagnosing hepatocellular carcinoma. Hepatogastroenterology. 2011;58(110-111):1718-1724.

11. Li B, Liu H, Shang HW, Li P, Li N, Ding HG. Diagnostic value of glypican-3 in alpha fetoprotein negative hepatocellular carcinoma patients. Afr Health Sci. 2013;13(3):703-709.

12. Liu JW, Zuo XL, Wang S. Diagnosis accuracy of serum glypican-3 level in patients with hepatocellular carcinoma and liver cirrhosis: a meta-analysis. Eur Rev Med Pharmacol Sci. 2015;19(19):3655-3673.

13. Sham JG, Kievit FM, Grierson JR, et al. Glypican-3-targeted Zr PET imaging of hepatocellular carcinoma. J Nucl Med. 2014;55(5): 799-804.

14. Zhu D, Qin Y, Wang J, et al. Novel Glypican-3-binding peptide for in vivo hepatocellular carcinoma fluorescent imaging. Bioconjug Chem. 2016; 27(3):831-839.

15. Fu SJ, Qi CY, Xiao WK, Li SQ, Peng BG, Liang LJ. Glypican-3 is a potential prognostic biomarker for hepatocellular carcinoma after curative resection. Surgery. 2013;154(3):536-544.

16. Yorita K, Takahashi N, Takai H, et al. Prognostic significance of circumferential cell surface immunoreactivity of glypican-3 in hepatocellular carcinoma. Liver Int. 2011;31(1):120-131.

17. Shirakawa H, Suzuki H, Shimomura M, et al. Glypican-3 expression is correlated with poor prognosis in hepatocellular carcinoma. Cancer Sci. 2009;100(8):1403-1407.

18. Li J, Gao JZ, Du JL, Wei LX. Prognostic and clinicopathological significance of glypican-3 overexpression in hepatocellular carcinoma: a meta-analysis. World J Gastroenterol. 2014;20(20):6336-6344.

19. Xiao WK, Qi CY, Chen D, et al. Prognostic significance of glypican-3 in hepatocellular carcinoma: a meta-analysis. BMC Cancer. 2014; 14:104.

20. Capurro MI, Xiang YY, Lobe C, Filmus J. Glypican-3 promotes the growth of hepatocellular carcinoma by stimulating canonical Wnt signaling. Cancer Res. 2005;65(14):6245-6254.

21. Li L, Jin R, Zhang X, et al. Oncogenic activation of GPC3 by c-Myc in human hepatocellular carcinoma. Hepatology. 2012;56(4):1380-1390.

22. Zittermann SI, Capurro MI, Shi W, Filmus J. Soluble glypican 3 inhibits the growth of hepatocellular carcinoma in vitro and in vivo. Int J Cancer. 2010;126(6):1291-1301.

23. Sun CK, Chua MS, He J, So SK. Suppression of glypican 3 inhibits growth of hepatocellular carcinoma cells through up-regulation of TGF-ß2. Neoplasia. 2011;13(8):735-747.

24. Gauglhofer C, Sagmeister S, Schrottmaier W, et al. Up-regulation of the fibroblast growth factor 8 subfamily in human hepatocellular carcinoma for cell survival and neoangiogenesis. Hepatology. 2011;53(3): 854-864.

25. Acloque H, Adams MS, Fishwick K, Bronner-Fraser M, Nieto MA. Epithelial-mesenchymal transitions: the importance of changing cell state in development and disease. J Clin Invest. 2009;119(6): 1438-1449.

26. Wu Y, Liu H, Weng H, et al. Glypican-3 promotes epithelial-mesenchymal transition of hepatocellular carcinoma cells through ERK signaling pathway. Int J Oncol. 2015;46(3):1275-1285. 
27. Ruan J, Liu F, Chen X, et al. Inhibition of glypican-3 expression via RNA interference influences the growth and invasive ability of the MHCC97-H human hepatocellular carcinoma cell line. Int $J$ Mol Med. 2011;28(4):497-503.

28. Baumhoer D, Tornillo L, Stadlmann S, Roncalli M, Diamantis EK, Terracciano LM. Glypican 3 expression in human nonneoplastic, preneoplastic, and neoplastic tissues: a tissue microarray analysis of 4,387 tissue samples. Am J Clin Pathol. 2008;129(6): 899-906.

29. Llovet JM, Chen Y, Wurmbach E, et al. A molecular signature to discriminate dysplastic nodules from early hepatocellular carcinoma in HCV cirrhosis. Gastroenterology. 2006;131(6):1758-1767.

30. Zhu ZW, Friess H, Wang L, et al. Enhanced glypican-3 expression differentiates the majority of hepatocellular carcinomas from benign hepatic disorders. Gut. 2001;48(4):558-564.

31. Ho M, Kim H. Glypican-3: a new target for cancer immunotherapy. Eur J Cancer. 2011;47(3):333-338.

32. Filmus J, Capurro M. Glypican-3: a marker and a therapeutic target in hepatocellular carcinoma. FEBS J. 2013;280(10):2471-2476.

33. Nakano K, Ishiguro T, Konishi H, et al. Generation of a humanized anti-glypican 3 antibody by CDR grafting and stability optimization. Anticancer Drugs. 2010;21(10):907-916.
34. Nakano K, Orita T, Nezu J, et al. Anti-glypican 3 antibodies cause ADCC against human hepatocellular carcinoma cells. Biochem Biophys Res Commun. 2009;378(2):279-284.

35. Ishiguro T, Sugimoto M, Kinoshita Y, et al. Anti-glypican 3 antibody as a potential antitumor agent for human liver cancer. Cancer Res. 2008;68(23):9832-9838.

36. Zhu AX, Gold PJ, El-Khoueiry AB, et al. First-in-man phase I study of GC33, a novel recombinant humanized antibody against glypican-3, in patients with advanced hepatocellular carcinoma. Clin Cancer Res. 2013;19(4):920-928.

37. Yen CJ, Daniele B, Kudo M, et al. Randomized phase II trial of intravenous RO5137382/GC33 at $1600 \mathrm{mg}$ every other week and placebo in previously treated patients with unresectable advanced hepatocellular carcinoma. J Clin Oncol. 2014;32(Suppl 5):abstract 4102.

38. Feng M, Ho M. Glypican-3 antibodies: a new therapeutic target for liver cancer. FEBS Lett. 2014;588(2):377-382.

39. Gao W, Tang Z, Zhang YF, et al. Immunotoxin targeting glypican-3 regresses liver cancer via dual inhibition of Wnt signalling and protein synthesis. Nat Commun. 2015;11(6):6536.

40. Gao H, Li K, Tu H, et al. Development of T cells redirected to glypican-3 for the treatment of hepatocellular carcinoma. Clin Cancer Res. 2014;20(24):6418-6428.
Journal of Hepatocellular Carcinoma

\section{Publish your work in this journal}

The Journal of Hepatocellular Carcinoma is an international, peerreviewed, open access journal that offers a platform for the dissemination and study of clinical, translational and basic research findings in this rapidly developing field. Development in areas including, but not limited to, epidemiology, vaccination, hepatitis therapy, pathology and

\section{Dovepress}

molecular tumor classification and prognostication are all considered for publication. The manuscript management system is completely online and includes a very quick and fair peer-review system, which is all easy to use. Visit http://www.dovepress.com/testimonials.php to read real quotes from published authors. 